\section{Sistema endocanabinoide y desarrollo de esteatosis hepática}

\author{
CARINA VALENZUELA ${ }^{1, a}$, VALESKA CASTILLO ${ }^{2, b}$, ANA MARÍA RONCO $^{2, \mathrm{c}}$, \\ CAROLINA AGUIRRE ${ }^{2, \mathrm{~d}}$, SANDRA HIRSCH ${ }^{3, \mathrm{e}}$, MIGUEL LLANOS ${ }^{2, \mathrm{f}}$
}

\section{A role for the endocannabinoid system in hepatic steatosis}

The endocannabinoid system (SEC) is an important modulator of several metabolic functions. This system is composed by cannabinoid receptors type 1 and 2 ( $R C B_{1}$ and $\left.R C B_{2}\right)$, their endogenous ligands, known as endocannabinoids, and the enzymes involved in their synthesis and degradation. A deregulated SEC originates metabolic alterations in several tissues, resulting in the typical manifestations of the metabolic syndrome. Liver steatosis of different origins constitutes a physiopathological condition where an altered hepatic SEC is observed. In this condition, there is an increased expression of $R C B_{1}$ and/or higher endocannabinoid levels in different hepatic cells, which may exert an autocrine/ paracrine hyperstimulation of $R C B_{1} / R C B_{2}$. Activation of $R C B_{1}$ stimulate the expression of several hepatocyte lipogenic factors, thus leading to increased de novo fatty acids synthesis and consequently to an abnormal accumulation of triglycerides. The effect of $\mathrm{RCB}_{2}$ activity on hepatic function is still controversial because, on one side its stimulation has an interesting protective effect on alcoholic liver disease while, on the other, it may enhance the development of hepatic steatosis in experimental models of diet-induced obesity. In this review we discuss the proposed mechanisms by which SEC is involved in the etiology of hepatic steatosis, as well as the therapeutic possibilities involving peripheral $R C B_{1} / R C B_{2}$ antagonism/agonism, for the treatment of this condition.

(Rev Med Chile 2014; 142: 353-360)

Key words: Endocannabinoids; Liver Diseases; Receptors Cannabinoid.

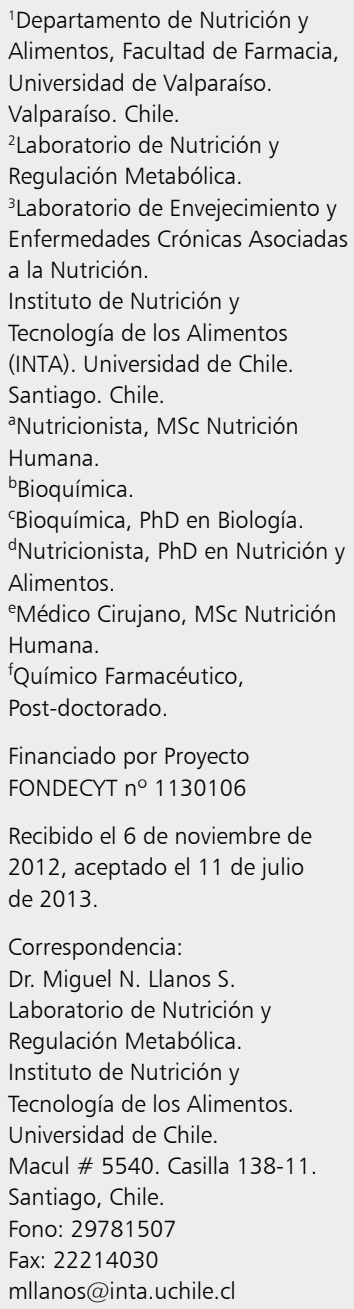

I

a esteatosis hepática se caracteriza por una acumulación excesiva de triglicéridos en los hepatocitos. Esta condición constituye el estado inicial de diversas enfermedades hepáticas, tales como enfermedad hepática por alcohol, enfermedad por hígado graso no alcohólica y hepatitis C. La progresión a un estado de cirrosis hepática deriva en complicaciones tales, que son de alto riesgo de mortalidad como la encefalopatía hepática, falla hepática por hipertensión portal y riesgo a desarrollar carcinoma hepatocelular. El daño hepático crónico es un importante problema de salud pública en la actualidad, con una mortalidad de alrededor de 1,4 millones de individuos/ año a nivel mundial ${ }^{1}$. En Chile, la Encuesta Nacio- nal de Salud 2009-2010 determinó que el riesgo de desarrollar daño hepático crónico por alcohol es de $39,1 \%$ en hombres y $25,2 \%$ en mujeres ${ }^{2}$, siendo la mortalidad total por cirrosis y otras enfermedades crónicas del hígado de 3.249 defunciones durante el año $2009^{3}$.

La acumulación excesiva de triglicéridos a nivel hepático se puede deber a distintos mecanismos: 1) aumento de la síntesis de novo de ácidos grasos; 2) disminución de la oxidación de ácidos grasos; 3) transporte aumentado de ácidos grasos desde tejidos periféricos hacia el hígado; 4) bloqueo del transporte de ácidos grasos desde el hígado a la circulación general y a tejidos periféricos. Si esta condición patológica persiste, progresa a infla- 
mación, fibrosis y finalmente cirrosis hepática, especialmente en presencia del virus de la hepatitis C, diabetes y obesidad.

Existe evidencia reciente que implica al sistema endocanabinoide (SEC) en la regulación de la hemodinamia hepática, fibrogénesis y metabolismo lipídico, además de su participación en la desregulación de estas funciones en estados patológicos, como en la esteatosis hepática de origen alcohólico o dietario. Por lo tanto, es necesario discernir los mecanismos implicados en el desarrollo de estas patologías, como una estrategia potencial para el tratamiento del daño hepático a través de la modulación del SEC.

\section{Sistema endocanabinoide. Características generales}

El sistema endocanabinoide lo comprenden fundamentalmente los receptores tipo 1 y 2 para canabinoides $\left(\mathrm{RCB}_{1}\right.$ y $\left.\mathrm{RCB}_{2}\right)$, sus agonistas endógenos, siendo los más estudiados la anandamida (ADA) y el 2-araquidonilglicerol (2-AG), y las enzimas responsables de su formación y degradación $n^{4,5}$.

Los endocanabinoides ADA y 2-AG, son biosintetizados a partir de fosfolípidos de membrana. La ADA se une con gran afinidad al $\mathrm{RCB}_{1}$ y con menor selectividad a $\mathrm{RCB}_{2}$. En tanto, el 2-AG se une casi con la misma afinidad a ambos receptores ${ }^{6}$ (Figura 1). Luego de ejercer su acción, la ADA es degradada específicamente por la acción de la enzima ácido graso amido hidrolasa (AGAH), mientras que el 2-AG es inactivado por la enzima monoacilglicerol lipasa (MAGL).

Los $\mathrm{RCB}_{1}$ y $\mathrm{RCB}_{2}$ son receptores de membrana acoplados a proteína $G$, inicialmente descubiertos como blancos moleculares del (-)-D ${ }^{9}$ tetrahidrocannabinol ( $\mathrm{D}^{9}$-THC), el componente psicotrópico de la planta Cannabis Sativa. Dichos receptores participan en la modulación fisiológica de varias funciones del sistema nervioso central y de diversos tejidos periféricos al ser activados por sus agonistas endógenos. $\mathrm{El} \mathrm{RCB}_{1}$ se expresa tanto a nivel central como periférico (tracto gastrointestinal, hígado, páncreas, músculo esquelético, tejido adiposo, etc. $)^{7}$; en tanto que el $\mathrm{RCB}_{2}$ se expresa principalmente en las células del sistema hematopoyético e inmune ${ }^{8}$. Sin embargo, en los últimos años se ha descrito la presencia del $\mathrm{RCB}_{2}$ en cerebro ${ }^{9}$ y en tejidos periféricos tales como páncreas e hígado ${ }^{10,11}$ jugando un rol importante en la funcionalidad de estos tejidos.

\section{SEC en hígado}

Hace menos de una década se estableció la presencia del SEC a nivel hepático, conociéndose cada vez más su participación en distintas funciones metabólicas inherentes a la fisiología y fisiopatología hepática. En condiciones normales, los receptores para canabinoides se expresan débilmente en el hígado ${ }^{12}$; observándose bajos niveles de $\mathrm{RCB}_{1}$ en hepatocitos y células endoteliales, y de

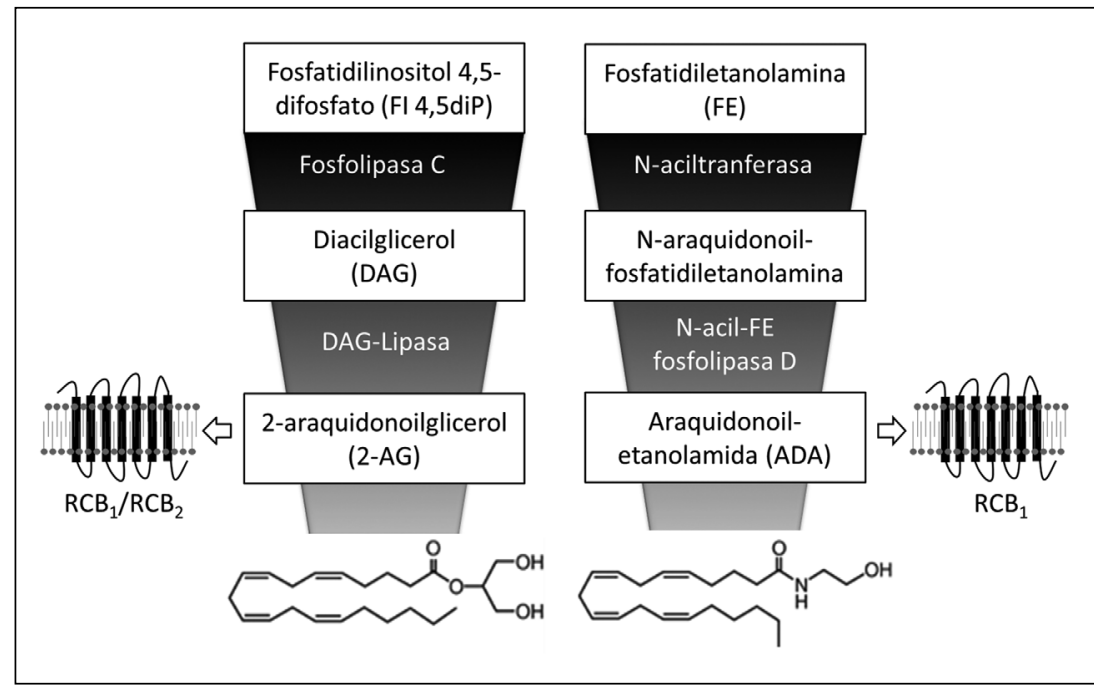

Figura 1. Rutas metabólicas vinculadas a la síntesis de los endocanabinoides Anandamida (ADA) y 2-Araquidonoilglicerol (2-AG) a partir de fosfolípidos de membrana. Luego de sintetizados, estos endocanabinoides son capaces de activar $\mathrm{RCB}_{1}$ y $\mathrm{RCB}_{2}$ estimulando diversos procesos metabólicos en tejidos blanco. 
$\mathrm{RCB}_{2}$ en células de Kupffer. Sin embargo, el daño hepático en distintos grados está asociado a un aumento de la expresión de RCB en hepatocitos, miofibroblastos y células endoteliales y de $\mathrm{RCB}_{2}$ en células de Kupffer y miofibroblastos ${ }^{1}$. Adicionalmente, en respuesta a daño hepático agudo o crónico hay un aumento de la concentración de 2-AG en hepatocitos y en células estrelladas, y de ADA en hepatocitos, células endoteliales y de Kupffer ${ }^{11,13,14}$. Estos antecedentes indican la existencia de un mayor tono endocanabinoide hepático en condiciones fisiopatológicas, tales como la esteatosis alcohólica y no alcohólica.

\section{SEC y esteatosis hepática no alcohólica}

El hígado graso no-alcohólico (HGNA) es la señal hepática del síndrome metabólico y es bastante común en la práctica clínica, con una prevalencia de $20-30 \%$ en países occidentales ${ }^{15}$. La manifestación de la enfermedad va desde una simple esteatosis a una esteatohepatitis, un estado que incluye esteatosis, inflamación, daño hepático y activación de fibrogénesis, con un riesgo de 10$20 \%$ de presentar cirrosis luego de 10 a 20 años ${ }^{16}$. El progreso de esteatosis a esteatohepatitis no está claramente dilucidado y parece ser de carácter multifactorial. Estudios recientes indican la participación de metabolitos lipotóxicos de ácidos grasos provenientes del tejido adiposo, como también el aumento de lipogénesis de novo en el hígado. El daño hepatocelular y su progresión es potenciado por la acción de citoquinas derivadas de macrófagos infiltrados ${ }^{17}$.

Tanto la obesidad en humanos como la inducida en modelos animales están a menudo asociadas a esteatosis hepática; sin embargo, ambas condiciones no ocurren en animales "knockout" para el gen que codifica a $\mathrm{RCB}_{1}\left(\mathrm{RCB}_{1}^{-/-}\right)^{18}$. Por otra parte, en ratas genéticamente obesas (Zucker) es posible reducir su cuadro de esteatosis luego de un tratamiento crónico con un antagonista/agonista inverso de $\mathrm{RCB}_{1}{ }^{19}$. En este sentido, los nuevos estudios se han enfocado en asociar la maquinaria molecular hepática con las acciones metabólicas reguladas por el SEC, que son conducentes al desarrollo de lipogénesis de novo. Aunque normalmente los hepatocitos expresan $\mathrm{RCB}_{1}$ en bajos niveles, al ser activados estos receptores estimulan la síntesis de novo de ácidos grasos a través de una secuencia de reacciones que implican un au- mento en la expresión del factor de transcripción conocido como proteína de unión del elemento regulador de esteroles 1c (SREBP-1c), que a su vez induce una mayor expresión de sus enzimas blanco: la acetil coenzima A carboxilasa-1 (ACC1) y la ácido graso sintasa (AGS) ${ }^{18}$. En forma concordante, no hay respuesta lipogénica del hígado en ratones $\mathrm{RCB}_{1}^{-1-}$; en tanto que ratones nativos tratados con un antagonista/agonista inverso de $\mathrm{RCB}_{1}$ (Rimonabant), muestran una disminución de la síntesis de novo de ácidos grasos hepáticos. $\mathrm{Al}$ respecto, resulta interesante el hallazgo que demuestra que una dieta alta en grasa es capaz de estimular la producción de ADA a nivel hepático y al mismo tiempo aumentar la expresión de $\mathrm{RCB}_{1}$ en hepatocitos ${ }^{18}$. Ambas condiciones indican la existencia de una hiperactivación de $\mathrm{RCB}_{1}$, derivando finalmente en un aumento de la síntesis de novo de ácidos grasos y la posterior acumulación de triglicéridos. Estudios más recientes, realizados en un modelo animal con una delesión hígadoespecífica de la expresión de $\mathrm{RCB}_{1}\left(\mathrm{hRCB}_{1}{ }^{-/}\right)$, demostraron que estos receptores hepáticos se requieren para el desarrollo de esteatosis inducida por dieta, dislipidemia y resistencia a insulina y leptina ${ }^{20}$. Además, estos animales $\mathrm{hRCB}_{1}^{-1-}$ alimentados con una dieta alta en grasa desarrollan obesidad, pero no presentan esteatosis hepática, ni dislipidemia ni resistencia a insulina. Estudios recientes en cultivos de hepatocitos demuestran además que la activación de $\mathrm{RCB}_{1}$ no solo estimula la lipogénesis de novo, sino que también reduce la oxidación de ácidos grasos y bloquea la liberación de las lipoproteínas de muy baja densidad (VLDL) ricas en triglicéridos. En este último caso, se ha demostrado que hay una rápida depleción del exceso de triglicéridos hepáticos luego de un bloqueo global de $\mathrm{RCB}_{1}$ periféricos en modelos animales de obesidad inducida por dieta ${ }^{21}$. Este hallazgo sugiere

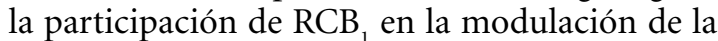
velocidad de secreción de VLDL desde hígado a la circulación sanguínea. El antagonista utilizado en este estudio, denominado AM6545, oralmente biodisponible y con una limitada penetración a nivel del sistema nervioso central, es capaz de revertir la esteatosis hepática no alcohólica asociada a obesidad y mejorar la dislipidemia ${ }^{21}$.

Otro receptor importante en la fisiopatología hepática es el receptor tipo 2 para canabinoides $\left(\mathrm{RCB}_{2}\right)$. Se ha descrito que los $\mathrm{RCB}_{2}$, que normalmente son casi indetectables en el hígado, 
están fuertemente inducidos en hepatocitos y colangiocitos en casos de esteatosis no alcohólica e hígado graso ${ }^{22}$. En cirrosis hepática declarada, dichos receptores se encuentran ampliamente distribuidos en el hígado, tanto en hepatocitos, células estrelladas, colangiocitos y miofibroblastos. Estos antecedentes sugieren la participación de $\mathrm{RCB}_{2}$ en los cambios metabólicos que caracterizan la fisiopatología hepática en diversos grados. En este sentido, ratones knockout para $\mathrm{RCB}_{2}\left(\mathrm{RCB}_{2}^{-/-}\right)$son resistentes a la esteatohepatitis inducida por dieta en comparación a los ratones nativos con igual alimentación. Adicionalmente, el tratamiento con un agonista de $\mathrm{RCB}_{2}$ incrementó más aún la acumulación de triglicéridos hepáticos en ratones nativos $^{23}$. Sin embargo, en la actualidad se requieren más estudios para dilucidar el rol del $\mathrm{RCB}_{2}$ en la patogénesis del hígado graso, como también la participación de otros posibles receptores canabinoides con una función aún desconocida en la biología hepática ${ }^{24}$ (Figura 2).

\section{SEC y esteatosis hepática alcohólica}

El alcoholismo, que puede conducir al desarrollo de hígado graso y progresar a cirrosis, es una de las principales causas de morbimortalidad relacionadas con daño hepático. El consumo de alcohol estimula la lipogénesis hepática e inhibe la oxidación de los ácidos grasos ${ }^{25}$. Teniendo en consideración que el mecanismo por el cual se induce esteatosis por alcohol y por dieta es similar (estimulación SREBP-1c) y que el alcohol induce un aumento de los niveles de endocanabinoides en cerebro $^{26}$, se ha sugerido que el SEC participa también en el desarrollo de la esteatosis alcohólica. De hecho, modelos en ratones con ingesta alcohólica crónica mostraron que la esteatosis hepática está asociada a un aumento en la expresión de $\mathrm{RCB}_{1} \mathrm{y}$ niveles de 2-AG en células estrelladas; condición que se puede evitar con la administración de un antagonista de $\mathrm{RCB}_{1}{ }^{13}$. Los niveles aumentados de 2-AG generan una hiperestimulación paracrina de los $\mathrm{RCB}_{1}$ presentes en los hepatocitos adyacentes, induciendo la sobre-expresión de genes lipogénicos y disminuyendo la excreción de triglicéridos hepáticos y la oxidación de ácidos grasos. Resultados adicionales indican que ratones nativos tratados con un antagonista de $\mathrm{RCB}_{1}$ y aquellos $\mathrm{RCB}_{1}^{-/-}$y hRCB ${ }_{1}^{-/-}$, son resistentes al desarrollo de hígado graso inducido por la ingesta de alcohol ${ }^{13}$.

Por otra parte, se ha sugerido que la activación de los $\mathrm{RCB}_{2}$ podría ser un nuevo objetivo terapéutico en las enfermedades hepáticas por abuso de alcohol. En un modelo de ratas cirróticas, el agonista de $\mathrm{RCB}_{2}$, JWH-133, mejora la respuesta regenerativa a una lesión hepática aguda y disminuye la fibrosis ${ }^{27}$. La activación de $\mathrm{RCB}_{2}$ también puede tener efectos beneficiosos sobre

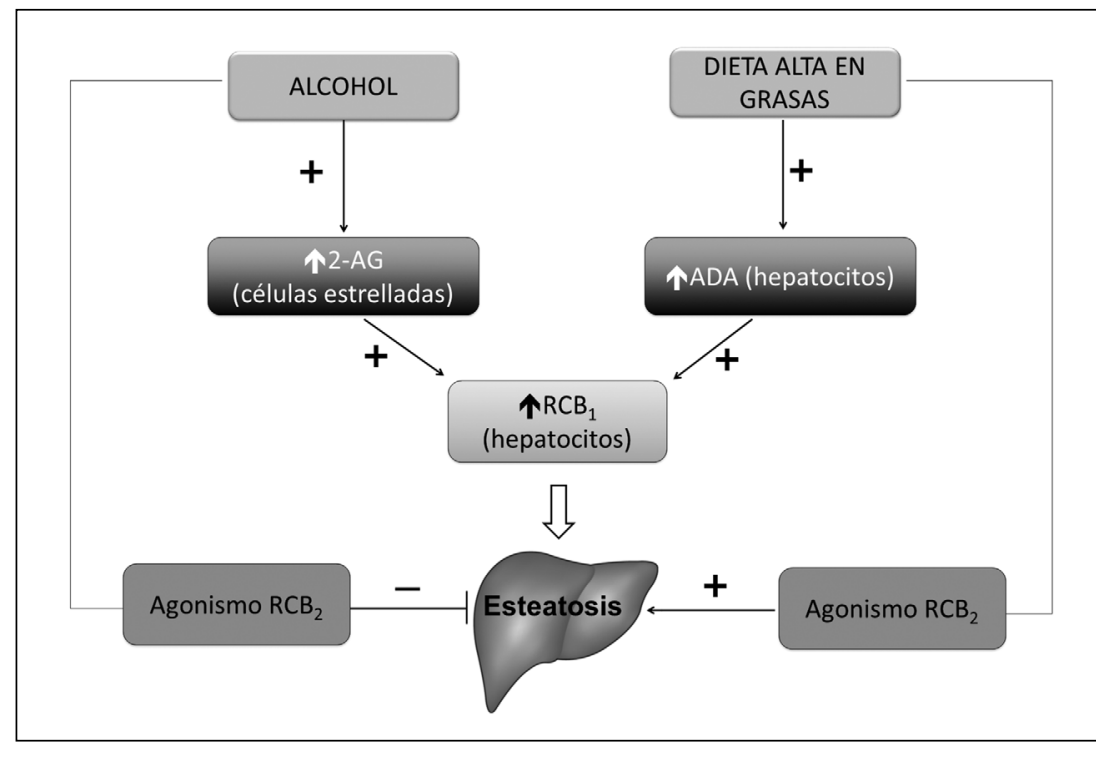

Figura 2. Participación del sistema endocanabinoide en la etiología de la esteatosis hepática. Es importante notar los efectos opuestos del agonismo de RCB en relación a la causa de esta patología; ya sea por consumo de dieta obesogénica o alcohol. 
el daño hepático debido a hepatitis inducida por tetracloruro de carbono $\left(\mathrm{CCl}_{4}\right)$, ya que promueve regeneración tisular vía mecanismos paracrinos que inicialmente implican la participación de miofibroblastos hepáticos ${ }^{28}$. La enzima inducible sintetasa de óxido nítrico (iSON) se ha identificado como la molécula clave capaz de mediar los efectos beneficiosos de $\mathrm{RCB}_{2}$ en hígado ${ }^{28}$, ya que ratones deprivados de la expresión de $\mathrm{RCB}_{2}$ $\left(\mathrm{RCB}_{2}^{-/-}\right)$muestran una inducción disminuida de la iSON hepática cuando son estimulados con $\mathrm{CCl}_{4}$; en tanto que ratones deprivados de expresión de iSON muestran un aumento de la apoptosis del hepatocito cuando son expuestos a los efectos deletéreos de dicho solvente orgánico ${ }^{29}$.

Otros estudios indican que la activación de $\mathrm{RCB}_{2}$ reduce la acumulación de triglicéridos en hepatocitos de ratones expuestos a una ingesta crónica de alcohol ${ }^{30}$. Adicionalmente, estudios en ratones $\mathrm{RCB}_{2}^{-/-}$expuestos a alcohol presentan una esteatosis hepática exacerbada, mientras que animales nativos tratados con un agonista selectivo para $\mathrm{RCB}_{2}$ (JWH-133) no desarrollan esteatosis por ingesta alcohólica. Dado que los $\mathrm{RCB}_{2}$ no se expresan en los hepatocitos ${ }^{23,28}$, los datos sugieren que la señal anti-esteatogénica se podría originar en las células de Kupffer. Estos antecedentes sugieren que la activación de $\mathrm{RCB}_{2}$ tiene un efecto beneficioso en el daño hepático por alcohol, limitando la inflamación y la esteatosis a través de efectos autocrinos y paracrinos. En este sentido, experimentos in vitro han confirmado que la activación de $\mathrm{RCB}_{2}$ regula la polarización de los macrófagos, previniendo la respuesta proinflamatoria M1 e induciendo la respuesta antiinflamatoria $\mathrm{M} 2^{30}$, lo que reduce la acumulación de grasa en el hígado.

\section{Evidencia clínica}

En relación a algunos estudios clínicos, estos coinciden con los datos preclínicos descritos anteriormente. Se sabe que pacientes obesos con tendencia a desarrollar síndrome metabólico poseen un tono endocanabinoide aumentado, teniendo niveles séricos de 2-AG mayores que los que presentan los individuos delgados ${ }^{31,32}$. Datos de la literatura indican que hay una correlación positiva entre las concentraciones de $2-\mathrm{AG}$ en sangre arterial y venosa hepática y el contenido de lípidos en hígado ${ }^{33}$. Adicionalmente, un estudio reciente demostró un robusto aumento de $\mathrm{RCB}_{\text {hepáticos }}$ en individuos con hígado graso no alcohólico ${ }^{34}$. El impacto del SEC sobre la esteatosis hepática se ha investigado más acuciosamente en pacientes con hepatitis $\mathrm{C}$, en los cuales la prevalencia de esteatosis es de $30-70 \%$, ya sea como consecuencia de perturbaciones metabólicas asociadas o bien por los efectos esteatogénicos propios del genotipo 3 del virus de la hepatitis $C$. En un estudio prospectivo en pacientes con hepatitis $\mathrm{C}$ no tratados, el uso diario de Cannabis durante 6 meses se reconoció como un predictor independiente de esteatosis severa $^{35}$. Adicionalmente, se ha demostrado una correlación positiva entre el nivel de expresión de $\mathrm{RCB}_{1}$ hepáticos y el grado de severidad de la esteatosis por hepatitis $\mathrm{C}^{36}$.

Aunque los estudios clínicos han sido suspendidos en la actualidad debido a los efectos indeseables de los antagonistas de $\mathrm{RCB}_{1}$ a nivel del sistema nervioso central, evidencias previas e indirectas sugieren que el bloqueo de $\mathrm{RCB}_{1}$ podría haber sido una aproximación válida para el manejo de la esteatosis hepática no-alcohólica. El análisis de los datos obtenidos de cuatro estudios independientes en pacientes con sobrepeso, indica una disminución de los niveles séricos de alanina aminotransferasa (ALT) en aquellos sujetos bajo tratamiento con rimonabant en relación a los tratados con un placebo, lo que sugiere un impacto beneficioso sobre el hígado graso ${ }^{37}$. Adicionalmente, el ensayo ADAGIO-Lípidos, en alrededor de 800 pacientes con obesidad abdominal y dislipidemia, reportó resultados similares ${ }^{38}$. Más aun, un subestudio dentro del ensayo descrito anteriormente, desarrollado para evaluar depósitos lipídicos hepáticos por tomografía computarizada, reveló una disminución de la esteatosis hepática en los pacientes tratados con rimonabant versus los pacientes sometidos a placebo. Todas estas evidencias indican la participación de los endocanabinoides y de los RCB ${ }_{1}$ hepáticos en el desarrollo de esteatosis; y por lo tanto validan la posibilidad de inhibir este receptor en el tratamiento de esta condición patológica; aun cuando dicha inhibición debiese estar restringida a nivel periférico para evitar los efectos secundarios asociados a un impacto indeseable en la función cerebral ${ }^{6,39,40}$.

Finalmente, es necesario recordar que rimonabant se utilizó terapéuticamente en varios países, fundamentalmente en Europa, durante alrededor de dos años (2006-2008), período en el que se 
constató que este compuesto, al inhibir los $\mathrm{RCB}_{1}$ presentes en el sistema nervioso central, generaba problemas de ansiedad y depresión marcada en pacientes susceptibles ${ }^{37,41}$. El SEC está involucrado en la mantención de un estado homeostático de comportamiento, respondiendo a períodos de estrés y ansiedad a través de una modulación neuronal retrógrada ${ }^{42,43}$ debido a la activación de $\mathrm{RCB}_{1}$ por sus agonistas endógenos $\mathrm{ADA}$ y $2-\mathrm{AG}^{44}$. De manera que el bloqueo de $\mathrm{RCB}_{1}$ en el sistema nervioso central altera los mecanismos de "manejo" del estrés y la ansiedad, facilitando la manifestación de efectos secundarios de carácter depresivo. Dado que los riesgos de desórdenes siquiátricos a causa de este compuesto sobrepasaban los efectos beneficiosos, la Agencia Europea de Medicina recomendó en octubre de 2008 la suspensión de la venta de este medicamento ${ }^{41,45}$. Por esta razón, en los últimos años, la investigación en esta área se ha enfocado en el desarrollo de compuestos que no atraviesen la barrera hemato-encefálica, ya sea antagonistas como agonistas inversos, de modo que ejerzan su acción de bloqueo de $\mathrm{RCB}_{1}$ solamente en órganos periféricos ${ }^{39,46,47}$. El potencial terapéutico de este tipo de compuestos sería de gran utilidad en el tratamiento de ciertas condiciones fisiopatológicas asociadas a la hiperactivación de $\mathrm{RCB}_{1}$, como por ejemplo las alteraciones hepáticas descritas en este artículo.

\section{Conclusiones}

En los últimos años se ha considerado que los endocanabinoides, a través de la activación de $\mathrm{RCB}_{1}$ hepáticos, han emergido como importantes moduladores del desarrollo de hígado graso, tanto el inducido por dieta como por alcohol. Ambas situaciones dan cuenta de la mayoría de los casos de cirrosis en las sociedades occidentales, lo que promueve el potencial uso terapéutico de antagonistas de $\mathrm{RCB}_{1}$ para el tratamiento de estas condiciones patológicas. Dados los efectos secundarios indeseables a nivel del sistema nervioso central reportados al utilizar rimonabant para el tratamiento de sobrepeso/obesidad (un antagonista/agonista inverso de $\mathrm{RCB}_{1}$ ), se ha formulado actualmente una segunda generación de antagonistas y agonistas inversos de este receptor $\mathrm{CB}_{1}$, con acción restringida a nivel periférico, que podrían ser útiles para el tratamiento de la esteatosis hepática de distinto origen.
Por otra parte, la posibilidad de utilizar agonistas de $\mathrm{RCB}$, sin efectos psicoactivos es un hecho plausible, dado los beneficios terapéuticos que puede presentar la activación de $\mathrm{RCB}_{2}$ en atenuar el daño hepático y promover la reparación del tejido en casos de fibrosis hepática por alcohol.

Finalmente, es importante considerar que el SEC hepático no sólo participa en la fisiología y fisiopatología del hígado, sino que también está implicado en otros efectos sistémicos asociados al síndrome metabólico, como es el caso de la dislipidemia y resistencia a insulina y leptina ${ }^{20,34}$.

Agradecimientos: Se agradece el apoyo financiero del Fondo Nacional de Ciencia y Tecnología de Chile, a través del proyecto ${ }^{\circ} 1130106$.

\section{Referencias}

1. Mallat A, Lotersztajn S. Cannabinoid receptors as therapeutic targets in the management of liver diseases. Drug News Perspect 2008; 21 (7): 363-8.

2. Encuesta Nacional de Salud 2009-2010, MINSAL Chile. [en línea: http://www.minsal.gob.cl/portal/url/item/ bcb03d7bc28b64dfe040010165012d23.pdf].

3. Estadísticas vitales, informe anual 2010, INE [en línea: http://www.ine.cl/canales/chile_estadistico/ demografia_y_vitales/estadisticas_vitales/pdf/vitales_ 2010.pdf].

4. Devane WA, Dysarz FA 3rd, Johnson MR, Melvin LS, Howlett AC. Determination and characterization of a cannabinoid receptor in rat brain. Mol Pharmacol 1988; 34: 605-13.

5. Devane WA, Hanus L, Breuer A, Pertwee RG, Stevenson LA, Griffin $\mathrm{G}$, et al. Isolation and structure of a brain constituent that binds to the cannabinoid receptors. Science 1992; 258: 1946-9.

6. Valenzuela C, Aguirre C, Castillo V, Ronco AM, Llanos M. [A role for the endocannabinoid system in obesity]. Rev Med Chile 2010; 138 (5): 621-9.

7. Matias I, Petrosino S, Racioppi A, Capasso R, Izzo AA, Di Marzo V. Dysregulation of peripheral endocannabinoid levels in hyperglycemia and obesity: Effect of high fat diets. Mol Cell Endocrinol 2008; 16: 286 (1-2 Suppl 1): S66-78.

8. Graham ES, Angel CE, Schwarz LE, Dunbar PR, Glass M. Detailed Characterisation of $\mathrm{CB} 2$ receptor protein expression in peripheral blood immune cells from healthy human volunteers using flow cytometry. Int J Immunopathol Pharmacol 2010; 23 (1): 25-34.

9. Callén L, Moreno E, Barroso-Chinea P, Moreno-Delgado 
D, Cortés A, Mallol J, et al. Cannabinoid receptors CB1 and CB2 form functional heteromers in brain. J Biol Chem 2012; 287 (25): 20851-65.

10. Juan-Picó P, Fuentes E, Bermúdez-Silva FJ, Javier Díaz-Molina F, Ripoll C, Rodríguez de Fonseca F, et al. Cannabinoid receptors regulate $\mathrm{Ca}^{2+}$ signals and insulin secretion in pancreatic beta-cell. Cell Calcium 2006; 39 (2): 155-62.

11. Bátkai S, Osei-Hyiaman D, Pan H, El-Assal O, Rajesh $\mathrm{M}$, Mukhopadhyay $\mathrm{P}$, et al. Cannabinoid-2 receptor mediates protection against hepatic ischemia/reperfusion injury. FASEB J 2007; 21 (8): 1788-800.

12. Mukhopadhyay B, Liu J, Osei-Hyiaman D, Godlewski G, Mukhopadhyay P, Wang L, et al. Transcriptional regulation of cannabinoid receptor-1 expression in the liver by retinoic acid acting via retinoic acid receptor-gamma. J Biol Chem 2010; 285 (25): 19002-11.

13. Jeong WI, Osei-Hyiaman D, Park O, Liu J, Bátkai S, Mukhopadhyay $\mathrm{P}$, et al. Paracrine activation of hepatic CB1 receptors by stellate cell-derived endocannabinoids mediates alcoholic fatty liver. Cell Metab 2008; 7 (3): 227-35.

14. Mallat A, Teixeira-Clerc F, Deveaux V, Manin S, Lotersztajn $\mathrm{S}$. The endocannabinoid system as a key mediator during liver diseases: new insights and therapeutic openings. Br J Pharmacol 2011; 163 (7): 1432-40.

15. Cortez-Pinto H, de Moura MC, Day CP. Non-alcoholic steatohepatitis: from cell biology to clinical practice. J Hepatol 2006; 44 (1): 197-208.

16. Ong JP, Younossi ZM. Epidemiology and natural history of NAFLD and NASH. Clin Liver Dis 2007; 11 (1): 1-16.

17. Tilg $\mathrm{H}$, Moschen AR. Evolution of inflammation in nonalcoholic fatty liver disease: the multiple parallel hits hypothesis. Hepatology 2010; 52 (5): 1836-46.

18. Osei-Hyiaman D, DePetrillo M, Pacher P, Liu J, Radaeva $S$, Batkai S, et al. Endocannabinoid activation at hepatic CB1 receptors stimulates fatty acid synthesis and contributes to diet-induced obesity. J Clin Invest 2005; 115: 1298-305.

19. Gary-Bobo M, Elachouri G, Gallas JF, Janiak P, Marini P, Ravinet-Trillou C, et al. Rimonabant reduces obesityassociated hepatic steatosis and features of metabolic syndrome in obese Zucker fa/fa rats. Hepatology 2007; 46 (1): 122-9.

20. Osei-Hyiaman D, Liu J, Zhou L, Godlewski G, HarveyWhite J, Jeong W, et al. Hepatic CB1 receptor is required for development of diet-induced steatosis, dyslipidemia, and insulin and leptin resistance in mice. J Clin Invest 2008; 118: 3160-9.

21. Tam J, Vemuri VK, Liu J, Bátkai S, Mukhopadhyay B, Godlewski G, et al. Peripheral CB1 cannabinoid recep- tor blockade improves cardiometabolic risk in mouse models of obesity. J Clin Invest 2010; 120 (8): 2953-66.

22. Méndez-Sánchez N, Zamora-Valdés D, Pichardo-Bahena R, Barredo-Prieto B, Ponciano-Rodríguez G, BermejoMartínez L, et al. Endocannabinoid receptor CB2 in nonalcoholic fatty liver disease. Liver Int 2007; 27 (2): 215-9.

23. Deveaux V, Cadoudal T, Ichigotani Y, Teixeira-Clerc F, Louvet A, Manin S, et al. Cannabinoid CB2 receptor potentiates obesity-associated inflammation, insulin resistance and hepatic steatosis. PLoS One 2009; 4 (6): e5844.

24. Begg M, Pacher P, Bátkai S, Osei-Hyiaman D, Offertáler L, Mo FM, et al. Evidence for novel cannabinoid receptors. Pharmacol Ther 2005; 106 (2): 133-45.

25. You M, Matsumoto M, Pacold CM, Cho WK, Crabb DW. The role of AMP-activated protein kinase in the action of ethanol in the liver. Gastroenterology 2004; 127 (6): 1798-808.

26. Basavarajappa BS, Saito M, Cooper TB, Hungund BL. Stimulation of cannabinoid receptor agonist 2-arachidonylglycerol by chronic ethanol and its modulation by specific neuromodulators in cerebellar granule neurons. Biochim Biophys Acta 2000; 15; 1535 (1): 78-86.

27. Muñoz-Luque J, Ros J, Fernández-Varo G, Tugues S, Morales-Ruiz M, Alvarez CE, et al. Regression of fibrosis after chronic stimulation of cannabinoid CB2 receptor in cirrhotic rats. J Pharmacol Exp Ther 2008; 324 (2): 475-83.

28. Teixeira-Clerc F, Belot MP, Manin S, Deveaux V, Cadoudal T, Chobert MN, et al. Beneficial paracrine effects of cannabinoid receptor 2 on liver injury and regeneration. Hepatology 2010; 52 (3): 1046-59.

29. Aram G, Potter JJ, Liu X, Torbenson MS, Mezey E. Lack of inducible nitric oxide synthase leads to increased hepatic apoptosis and decreased fibrosis in mice after chronic carbon tetrachloride administration. Hepatology 2008; 47 (6): 2051-8.

30. Louvet A, Texeira-Clerc F, Chobert MN, Deveaux V, Pavoine C, Zimmer A, et al. Cannabinoid CB2 receptors protects against alcoholic liver disease by regulating Kupffer cell polarization in mice. Hepatology 2011; 54 (4): 1217-26.

31. Engeli S, Böhnke J, Feldpausch M, Gorzelniak K, Janke J, Bátkai S, et al. Activation of the peripheral endocannabinoid system in human obesity. Diabetes 2005; 54 : 2838-43.

32. Côté M, Matías I, Lemieux I, Petrosino S, Alméras N, Després JP, et al. Circulating endocannabinoid levels, abdominal adiposity and related cardiometabolic risk factors in obese men. Int J Obes (Lond) 2007; 31: 692-9. 
33. Westerbacka J, Kotronen A, Fielding BA, Wahren J, Hodson L, Perttilä J, et al. Splanchnic balance of free fatty acids, endocannabinoids, and lipids in subjects with nonalcoholic fatty liver disease. Gastroenterology 2010; 139 (6): 1961-71.

34. Liu J, Zhou L, Xiong K, Godlewski G, Mukhopadhyay B, Tam J, et al. Hepatic cannabinoid receptor-1 mediates diet-induced insulin resistance via inhibition of insulin signaling and clearance in mice. Gastroenterology. 2012; 142 (5): 1218-28.

35. Hézode C, Zafrani ES, Roudot-Thoraval F, Costentin C, Hessami A, Bouvier-Alias M, et al. Daily cannabis use: a novel risk factor of steatosis severity in patients with chronic hepatitis C. Gastroenterology 2008; 134 (2): 432-9.

36. Van der Poorten D, Shahidi M, Tay E, Sesha J, Tran K, McLeod D, et al. Hepatitis $\mathrm{C}$ virus induces the cannabinoid receptor 1. PLoS One 2010; 5(9).

37. Van Gaal LF, Pi-Sunyer X, Despre's JP, Mc Carthy C, Scheen AJ. Efficacy and safety of rimonabant for improvement of multiple cardiometabolic risk factors in overweight/obese patients: pooled 1-year data from the RIO program. Diabetes Care 2008; 31 (Suppl 2): S22940.

38. Després JP, Golay A, Sjöström L. Rimonabant in Obesity-Lipids Study Group. Effects on metabolic risk factors in overweight patients with dyslipidemia. N Engl J Med 2005; 353: 2121-34.

39. Kunos G, Osei-Hyiaman D, Bátkai S, Sharkey KA, Makriyannis A. Should peripheral CB(1) cannabinoid receptors be selectively targeted for therapeutic gain? Trends Pharmacol Sci 2009; 30: 1-7.
40. Valenzuela CA, Castillo VA, Aguirre CA, Ronco AM, Llanos $\mathrm{MN}$. The $\mathrm{CB}_{1}$ receptor antagonist SR141716A reverses adult male mice overweight and metabolic alterations induced by early stress. Obesity (Silver Spring) 2011; 19 (1): 29-35.

41. Christensen R, Kristensen PK, Bartels EM, Bliddal H, Astrup A. Efficacy and safety of the weight-loss drug rimonabant: a meta-analysis of randomised trials. Lancet 2007; 370 (9600): 1706-13.

42. Malcher-Lopes R, Di S, Marcheselli VS, Weng FJ, Stuart CT, Bazan NG, et al. Opposing crosstalk between leptin and glucocorticoids rapidly modulates synaptic excitation via endocannabinoid release. J Neurosci 2006; 26: 6643-50.

43. Malcher-Lopes R, Franco A, Tasker JG. Glucocorticoids shift arachidonic acid metabolism toward endocannabinoid synthesis: a non-genomic anti-inflammatory switch. Eur J Pharmacol 2008; 583: 322-39.

44. Di S, Malcher-Lopes R, Halmos KC, Tasker JG. Nongenomic glucocorticoid inhibition via endocannabinoid release in the hypothalamus: a fast feedback mechanism. J Neurosci 2003; 23 (12): 4850-7.

45. Jones D. End of the line for cannabinoid receptor 1 as an anti-obesity target? Nat Rev Drug Discov 2008; 7: 961-2.

46. Tam J, Cinar R, Liu J, Godlewski G, Wesley D, Jourdan T, et al. Peripheral cannabinoid-1 receptor inverse agonism reduces obesity by reversing leptin resistance. Cell Metab 2012; 16 (2): 167-79.

47. Tam J, Vemuri VK, Liu J, Bátkai S, Mukhopadhyay B, Godlewski G, et al. Peripheral CB1 cannabinoid receptor blockade improves cardiometabolic risk in mouse models of obesity. J Clin Invest 2010; 120 (8): 2953-66. 